\title{
Motivation-Based Approach To Behavior Prediction
}

\author{
Ismail Dagli \\ STZ Softwaretechnik \\ 73734 Esslingen \\ Tel: +49711 1741649 \\ Fax: +497111747054 \\ ismail.dagli@stz-softwaretechnik.de
}

\author{
Dr. Dirk Reichardt \\ DaimlerChrysler AG \\ RIC/AA HPC T728 \\ Tel: +49711 1741427 \\ Fax: +497111747054 \\ dirk.reichardt@daimlerchrysler.com
}

\begin{abstract}
The design of advanced driver assistance systems always aims at enabling the driver to master today's traffic in a more safe and comfortable way. For future assistance systems, the assisting system must be aware of the current overall traffic situation. In order to judge the risks in a situation, the assistance system must be able to predict the behavior of the traffic participants around. Taking into account all possible future situations for the next few seconds is a task which quickly produces a complexity that can hardly be handled.
\end{abstract}

Taking the human driver as a role model for its software-counterpart, we propose a new concept which aims at modeling anticipation by taking the motivations of drivers as a basis. Starting with a set of motivations typical for highway traffic, for example the navigation motivation of taking an exit or of changing lanes to keep a constant speed, concrete situation specific goals are derived. A planning component generates the possible and fulfillable plans for all vehicles in the scenario with respect to the set of goals. Then, the observed actions of the vehicles around are compared to these plans in order to derive a plausibility for the underlying intentions. Eventually, prediction is performed for plausible behaviors of vehicles, which are always based on a motivation that can be taken as an explanation for it.

First results are shown in simulation for highway exit scenarios. In the situations shown, a prediction for an upcoming lane change of a vehicle can be made just by the knowledge of pre-defined motivations and the observation of a sequence of actions.

\section{Introduction}

The challenging vision of our current research is to develop a scene understanding methodology for advanced assistance systems that can cope with complex traffic scenarios. The aim is that next generation driver assistance systems should be able to optimize their information, warning and control strategy by considering driver preferences and the overall traffic situation. As an example, early recognition of an intervening vehicle would help to adapt the control strategy of an ACC (Adaptive Cruise Control) system early enough to avoid that the safety distance is violated after the intervening vehicle has ranged into the gap. Obviously, a strict control strategy that only considers the distance to the front vehicle is not capable of handling such a situation appropriately. Therefore, it becomes more and more important that assistant systems are provided with a complete and consistent representation of the overall situation.

An important aspect which emerges from the illustrations here is the importance of early recognition of maneuvers of other vehicles. Hence, one important area of research is plan recognition and prediction that facilitates early recognition of dangerous situations in future.

\section{System overview}

Real-world traffic scenarios are complex and it is not possible to list descriptions of all scenarios occurring in real world traffic. The approach introduced here suggests that plan recognition is based on dynamic planning, requiring only a set of abstract motivations which are defined upfront by the designer.

Motivation-based plan recognition and prediction bases on the idea that driving behavior is strongly affected by the motivations of the driver and that these motivations can be - at least for highway traffic - formulated with a few sentences. Therefore, we assume that the drivers of all surrounding vehicles have a set of motivations, which are representative for their driving behavior. We further assume that the driver sets situation dependent goals according to the given motivations. After setting the goals, the driver develops plans to achieve the situation dependent goals and chooses the one with the highest probability of success, the highest convenience, or the minimum risk. The driver executes the plan, 
monitors the progress of the situation continuously and re-plans if the outcome becomes less attractive than in the initial situation.

The approach of motivation-based behavior recognition assigns each driver in the scene a pre-defined set of possible and relevant motivations (relevant for the assistance system). Given the motivations and the current situation, the system assigns possible goals to each of the drivers and creates possible plans according to the goals. The plan recognition modules take these plans and assigns plausibility hypotheses according to the current observations.

Plan recognition is one important aspect but early discovery of conflicts in plans of two or more vehicles that can lead to critical situations require also reliable prediction of situations in the future. Prediction as it is suggested here is performed in a possible world structure, where possible futures are expanded within a tree and each node that represents a possible future is associated with a plausibility. Figure 1 gives a general overview of the approach.

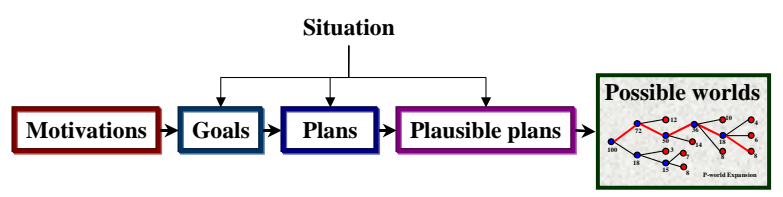

\section{Figure 1 Approach}

The expanded possible worlds are the basis of the situation analysis. Basically, situation analysis looks for relevant situations in the future that may affect the overall information, warning and control strategy of the assistance function. In case of ACC, situation analysis tries to discover situations where a vehicle crosses the own lane and forces an adaptation of the safety distance. In this work here we suggest that relevant terms for the assistance functions like road lanes or gaps are modeled as resources and situation analysis is responsible to discover conflicting operations on these resources over time and space.

The following sections will refine the illustrations given here. We will show how our system maps driving behavior into tangible terms to facilitate early recognition of critical situations. As a simplification we focus only on highway scenarios because those are less complex than scenarios given in city traffic.

\subsection{Motivations and goals}

Looking at the variety of actions that a vehicle can perform in a dynamically evolving traffic situation, it is very difficult to pre-define sequences or patterns of actions. Considering a complex traffic situation with many vehicles and taking all possible actions into account to predict future traffic situations would lead to combinatory explosion and is intractable, especially in real-time. Hence, instead of modeling fixed plans for recognition, we suggest to pre-define abstract motivations and to deduce goals and plans dynamically for all relevant vehicles in the scene. By considering only relevant and plausible (due to current observation) motivations prediction of future situations becomes feasible.

To make the discussion about motivations and goals more concrete we will introduce example motivations and goals especially for highway traffic. Given an arbitrary highway-traffic situation, we can assume a small set of abstract motivations for each vehicle in the scene. Please note that the following list shows only a sub-set of possible motivations that were used for test purposes in our application. Categories are arbitrarily chosen and must be refined in future work:

1. Timing motivation: $\mathrm{Be}$ at destination $\mathrm{x}$ at time $\mathrm{y}$. (This represents a timing requirement, from which, if occasion arises, we can deduce a higher trip velocity or an overtake intention.)

2. Personal motivation: Traffic law conform and safe driving.

3. Personal motivation: Dislike driving behind trucks.

\section{Navigation motivation: Drive from A to B.}

These motivation are dynamically mapped to goals. The following list represents only a sub-set of goals that can be deduced.

1. Drive at fast velocity. Overtake if necessary, e.g. if a slow vehicle is in front.

2. Drive at slow velocity. Prefer right lanes.

3. Overtake trucks and avoid driving on right most lane.

4. Find a safe path to exit lane or find a safe path to the highway, etc..

Deducing situation-dependent goals from motivations is a major task. In the first implementation, we classified situations in clusters and assigned abstract formulated goals to the set of motivations according to situation classes. Furthermore, we reduced the amount of motivations to those that are relevant for 
the application. For example, the four motivations given above have been tested for an ACC system and provided good coverage of intentions for a lane change in highway situation. Nevertheless, goal deduction remains a major area of research in order to find more scalable and general solutions.

\subsection{Plans}

To start, we decided to implement planning as situation space search [Russel, 1994]. We defined discrete operators which reflect the possible actions that a vehicle can perform in highway traffic. The situation is described in an abstract language in terms of vehicles, (time-)gaps, distances, velocities etc. The following figure shows the process from motivations to plans with the aid of an example. The example shows an "EGO" car that represents a vehicle equipped with our system. The "EGO" vehicle creates possible goals and plans for vehicle "A". The sequences of actions in the possible plans are represented with arrows to illustrate the discrete nature of operations/actions within the plan.

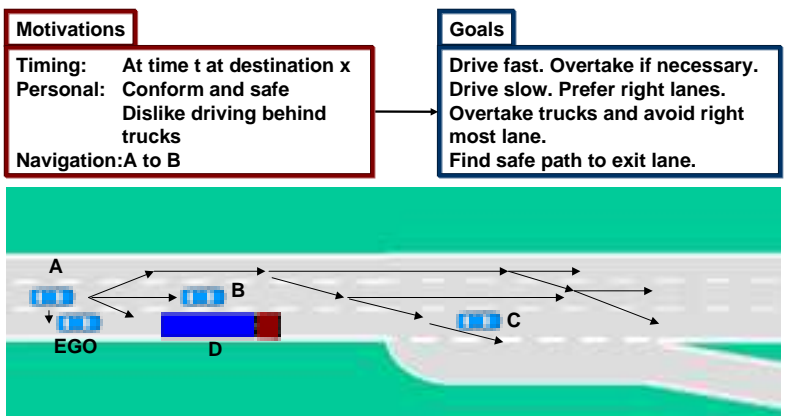

Figure 2 Motivations, goals and discrete plans

One of the motivations shown above would be to take the exit. For this motivation the goal deduction module derives a goal, according to the constraints given by the situation (constraints are derived from the environment data and the vehicle dynamics) :

$\begin{array}{ll}\text { Goalstate: } & \text { Exit Lane } \\ \text { Constraints: } & \text { StartExit Pos1 } \\ & \text { EndExit Pos2 } \\ & \text { Velocity } 15 \mathrm{~m} / \mathrm{s}\end{array}$

The planner uses a heuristic search to find a path from start state to goal state. The intuitive approach would be to define the value function $H(\vec{S})$ that rewards states that are closer to goal state. Therefore, a lane change to the right would preferred by the planner as a first step. This is of course a simple measure for plan quality and does not really reflect natural driving behavior. Therefore, we propose a more multi-dimensional measure, considering the following criteria:
Optimize time

Minimize lane changes

Minimize acceleration and deceleration

Minimize risk

Minimize distance to goal

Maximize safe distances to other vehicles

According to the assumed Motivations, one or more (weighted) criteria is used as a heuristic. Besides we define additional constraints like:

\section{Comply with vehicle dynamics}

Follow traffic rules

If the heuristic is defined appropriately the most realistic plan is generated first. The planner stops to generate possible plans after the last generated plan is below a certain reward threshold. Firstly, this restricts the amount of possible plans that is essential for efficiency purposes, and secondly it provides a measure that helps to assign plausibilites to plans in the plan recognition module (see section 2.3.2).

\subsection{Behavior recognition}

Actually, behavior recognition can be viewed as the reverse process of motivation-based driving behavior. Behavior recognition starts at the action level and tries to match sequences of actions to previously generated plans. The following paragraphs discuss the main issues involved in behavior recognition: action and plan recognition.

\subsubsection{Action recognition}

Action recognition is about tracking actions of vehicles in the scene and providing this information to the plan recognition components. An action within a plan transforms the agent from one plan state to the other. Although an action at the planning level is defined as a discrete operation, tracking of actions is preferably performed continuously to enable early recognition of the action. In this work here we define six possible actions which reflect the processes that a vehicle is able to perform. The first four actions are associated with the lateral and longitudinal movement and the last two modeled actions reflect that the observed vehicle neither performs a lateral nor longitudinal action ${ }^{1}$.

As an aggravating fact observation of actions are subject to uncertainty, and actions as modelled here, are not disjoint, by the means that two actions can occur in conjunction, observing a certain time relation. The time relations are modeled with the aid of Allen's temporal logic formalism [Allen 1984].

\footnotetext{
${ }^{1}$ Remaining also includes following and controlling
} 
Uncertainty is considered by using dynamic belief networks (DBN) for action recognition.

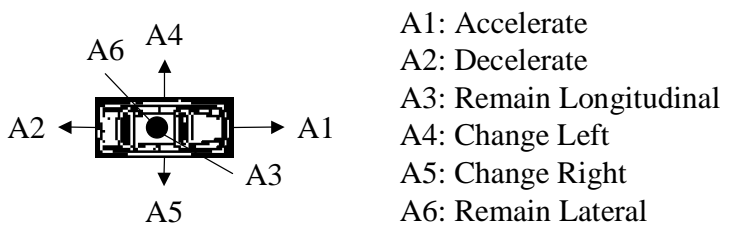

Figure 3 Actions

\subsubsection{Plan recognition}

Plan recognition takes the abstract plan specifications as input and assigns plausibility hypotheses to each possible plan of the observed vehicle. Plan recognition requires a history of actions to make hypotheses about the currently executed plan. Unfortunately, we re-plan at each cycle and discard formerly created plans, wherefore there is no history available. The consequence is that formerly assigned plausibility values are lost.

To overcome this problem we use two measures to assign appropriate plausibilities to plans. For plans that lead to the same goal, we assume that the plan with the highest value given by the heuristic search planner is the most plausible. Secondly, we map the history of actions to a goal plausibility. This is done by a general measure, which allows to compare plans leading to different goals according to their a priori plausibility. Hence, the whole history is mapped to a single goal plausibility value. Since the goals are more persistent than plans we achieve that plausibility measures of tracked plans can be transferred to the next planning cycle. To close the loop to motivation-based driving behavior, this scheme can be extended to motivations. Given the restricted observation horizon in a dynamic traffic situation it is clear that reasoning about plans, goals and motivations will not always be possible. We introduce inter-vehicle communication in the next section that is intended to assist the whole behavior recognition process.

\subsection{Integrating inter-vehicle communica- tion for plan recognition purposes}

CarTALK 2000 [Reichardt et. al. , 2001] is a new EC funded project focussing on new driver assistance systems which are based upon inter-vehicle communication. The idea is that inter-vehicle communication can be used to optimize current driver assistance systems by exchanging information about the current traffic situation. Inter-vehicle communi- cation is intended to augment the on-board sensor data and to facilitate new functionality such as hazard warning or co-operative driving.

In the context of plan recognition, inter-vehicle communication enables reliable transmission of intentions before the actual plan is executed. For example, a lane change intention or whole plans can be transmitted to all concerned vehicles in the scene, as an alternative to motivation-based plan recognition that is based on observation ${ }^{2}$. Alternatively, the vehicles could exchange motivations, so that the planning and plan recognition process can be focused and optimized.

Inter-vehicle communication assists the overall plan recognition process. Moreover, both exchange of maneuver intentions over inter-vehicle communication and the motivation based plan recognition that relies on the observation of the actions of vehicles can run in parallel to provide a degree of fault tolerance. It will be a very sophisticated and interesting task to combine both approaches in future projects.

\subsection{Possible worlds structure}

The possible worlds ${ }^{3}$ represent possible future situations given the plans of the vehicles. The structure is a tree that allows symbolic computation of future situations by combining possible actions of the vehicles in the scene. Considering combinations of all possible actions of all vehicles in the scene would obviously lead to combinatory explosion while creating possible worlds in future. Therefore, the prediction is concentrated on the plausible worlds given by the set of plausible plans.

We will illustrate the problem associated with computational complexity with the aid of a simple example. We first calculate all possible worlds given our notion about actions and plans and then try to reduce complexity with our approach. To simplify the calculations, we neglect the fact that actions can observe different time relations.

The computational complexity for calculating all possible worlds looking $\mathrm{T}$ steps in the future by

\footnotetext{
${ }^{2}$ Since we have driver in the loop, plan recognition must still be performed for the own driver

${ }^{3}$ In contrast to this notion here possible worlds are mostly associated with semantics to reason about own beliefs and beliefs of other agents and represent a popular semantics for modal logic. In this context, a possible world is an assignment of true or false to well-formed formulas of a theory. An accessible possible world is a world where all beliefs of ancestor worlds hold [Cresswell, 1999], [Dean, 1995].
} 
combining all possible actions of the agents is given by:

$\grave{E}\left(\sum_{n=1}^{T} \alpha^{\tau \times v}\right) \approx \grave{E}\left(\alpha^{\tau \times v}\right) \quad \alpha, \tau, v \in \mathrm{N}$

Formula 1

where $\alpha$ is the number of possible actions, $\tau$ is the time step in the future and $v$ is the number of vehicles in the scene. To predict possible situations for only five agents five steps long would require $\approx 3 \times 10^{19}$ possible worlds to be created. Contemplating that these worlds have to be created, stored and analyzed, it becomes pretty clear that more efficient ways have to be found.

Therefore, we defined the notion of plausible worlds and assigned computational resources according to the plausibility. The idea is to consider only valid and plausible plans for prediction, and to restrict the horizon of prediction according to the given plan plausibilities.

To follow the path given by the combination of the most plausible plans of all vehicles would only require that $T$ situations are created (most plausible path). If we were very sure about the plan plausibilities, this would be sufficient. But plausibility measures can not be assigned with full certainty. Therefore we need to consider a set of plausible futures.

In our approach, each possible world is assigned a plausibility value, given by the plan plausibilities. Assuming that motivations and also the plans of each vehicle are independent, the plausibility of each world can be calculated with:

$$
P l\left(\omega_{\tau+1}\right)=P l\left(\omega_{\tau}\right) \times \prod_{\substack{i j \mid i \in \text { plans, } \\ j \in \text { vehicles, } \\ \alpha_{\tau \mid i j} \in T\left(\omega_{\tau}, \omega_{\tau+1}\right)}} \operatorname{Pl}\left(\alpha_{\tau \mid j]} \mid \omega_{\tau}\right)
$$

Formula 2

Where $\operatorname{Pl}\left(\varpi_{\tau}\right)$ represents the plausibility of the current world in the possible world structure and $\operatorname{Pl}\left(\varpi_{\tau+1}\right)$ represents the plausibility of the following world. World $\varpi_{\tau+1}$ results from the combination of actions of the vehicles in $\varpi_{\tau}$. This set of actions is described by the transition function $\mathrm{T}\left(\varpi_{\tau}, \varpi_{\tau+1}\right)$. $\operatorname{Pl}\left(\mathrm{a}_{\tau \mid i j} \mid \varpi_{\tau}\right)$ is the plausibility of an action which is determined by the plan plausibilities in $\varpi_{\tau}$. Therefore, a path in the possible world structure gives a possible future when each vehicle follows one specified plan provided by the planner. Formula 2 facilitates to assign plausibility measures to a world in the possible world structure derived from the plan plausibilities given by the plan recognition modules.

Thus, it is only required to combine plans and not actions, which helps to focus the prediction step. Finally, we define a plausibility threshold $\delta$ to restrict the number of prediction steps dynamically. Given this threshold we expand paths in the possible world structure only if a possible world exceeds this threshold. Figure 4 illustrates the possible worlds structure where each node represents a world $\varpi_{\tau}$ along with a plausibility value. The longest path within the structure contains the worlds given by the combination of the most plausible plans of each vehicle.

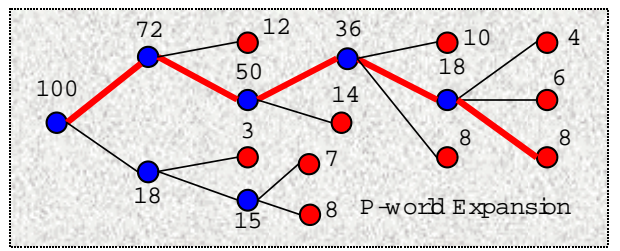

Figure 4 Possible worlds structure

Such a scheme to prediction enables dynamic assignment of computational resources. By setting a high threshold the computing time for prediction is restricted, with the result that prediction is performed only for few time steps.

\subsection{Situation analysis}

Situation analysis is carried out in this possible world structure. For ACC improvement we look for situations in the possible worlds where a vehicles crosses the lane of the vehicle equipped with our system. That means we look for patterns in situations and the evolution of situations that have the violation of the safety distance as consequence. The resulting Time-To-Collision is taken as risk measure. The system reacts if a risk threshold is exceeded within a very plausible world.

\section{Integrating planning and plan recogni- tion}

Figure 5 shows the resulting software architecture. Situations and Motivations are stored in a global database. The whole world representation consist of a history of situations, motivations, goals, plans and the possible worlds. Objects that represent the situation are fed into the world representation from the sensor and object fusion components. The results (P-Worlds) serve as basis for situation analysis. 


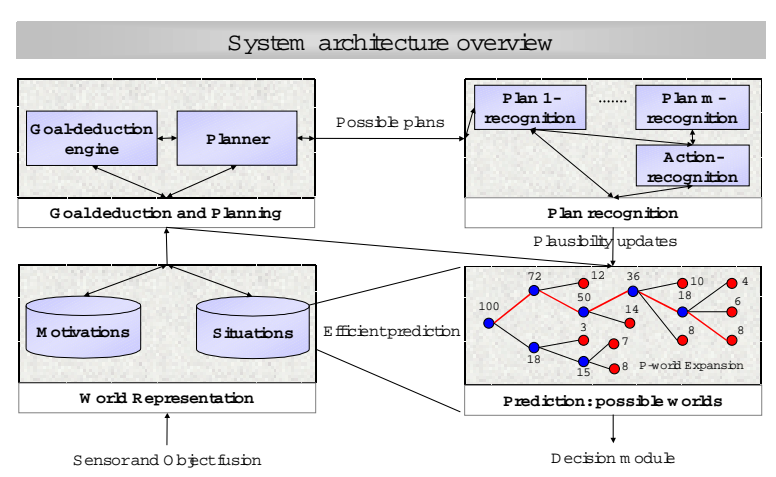

Figure 5 Architecture overview

Figure 6 shows the data and control flow of the plan recognition and prediction process. The process is performed at each clock cycle. Each world is assigned a risk value in the possible worlds structure which then serves a basis for the decision process of the information, warning or control system.

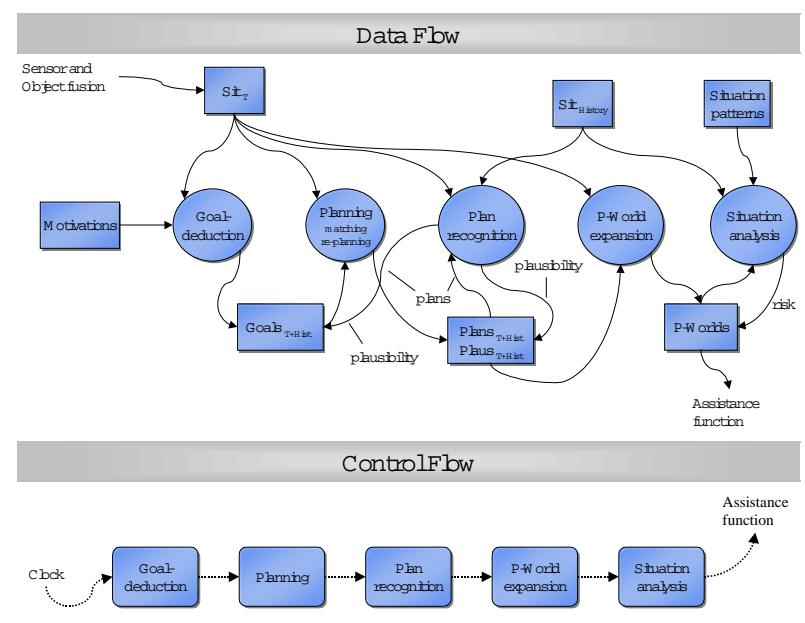

Figure 6 Data and control flow

\section{Simulation results}

Simulations have been carried out for various highway exit scenarios. The scenarios aimed at testing the ACC assistance system functionality. The ACC had been extended with behavior recognition and prediction capabilities as introduced here and the results were compared with the results of the ACC system without these capabilities.

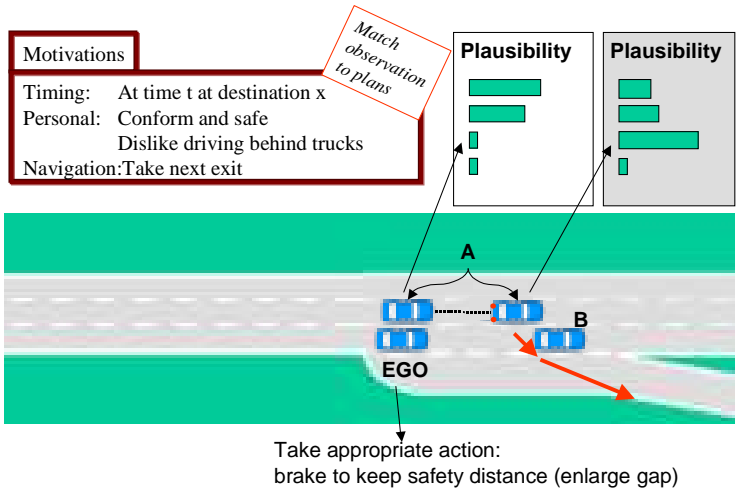

Figure 7 Simulated scenarios

In the first implementation, we tested the plan recognition and behaviour prediction capabilities, without using a dynamic planner (subject of further research). The aim was to validate the idea of behavior prediction by recognition and combination of plans of different vehicles. We especially focused on testing how this prediction capability enhances the overall driver assistance functions.

For this initial system, the prediction capability enabled early adaptation of the control strategy and improved the comfort properties of the system for a set of exit scenarios.

The system was able to anticipate a lane changing vehicle that crosses the own lane before the action actually occurred. The next major research topic will be the integration of a dynamic planning component into the overall system to cope with the variety of real world scenarios.

\section{Conclusion and outlook}

Preventing accidents and improving driving comfort are two major aims of driver assistance systems. This requires to look beyond reactivity and control. Next generation assistance systems should be able to imitate higher level human skills as planning of behavior or prediction of critical situations. The approach introduced here captures higher level skills associated with driving and puts them into tangible terms like motivations, goals, plans or prediction.

As soon as we reason about complex human behavior the problem gets computationally intractable. Especially, prediction and assessment of future situations is critical in terms of computation time and storage space. Therefore, it is essential to concentrate resources and to focus on plausible and relevant information.

Although concepts for plan recognition and prediction brought good results in simulation, we currently 
seek for more general and scalable solutions. Currently, we develop a dynamic planner and investigate how situations must be represented to allow appropriate situation analysis. Besides, we investigate situation pattern for appropriate goal deduction to enable the flexible approach here. Hence, most of areas introduced here are subject to further research to investigate the overall feasibility of such an approach. In a second step we will investigate how this approach can cope with limited sensor range and unreliable sensor information.

\section{References}

[Allen 1984] J.F. Allen, ,Towards a general Theory of Action“, In Artificial Intelligence, 23:123-154, 1984

[Cresswell, 1999] Max Cresswell, "Possible worlds semantics", The MIT encyclopedia of cognitive sciences, p. 659-661, 1999

[DEAN, 1995] Thomas Dean, James Allen, Yiannis Aloimnonds. "Artificial Intelligence. Theory and Practice", Addison \& Wesley, 1995.

[Halpern, 2001] J. Y. Halpern. "Plausibility Measures: A general approach for representing uncertainty“. Cornell University, Department of Computer Science, 2001.

[Reichardt et. al. , 2002] Dirk Reichardt, "CarTALK 2000 - Safe and comfortable driving based upon Inter-Vehicle Communication. Intelligent Vehicles, 2002.

[Russel, 1994] S.J. Russel, P.Norwig. "Artificial Intelligence, A Modern Approach". Prentice Hall, 1994. 